\title{
Endothelial CFTR dysfunction and its involvement in the pathogenesis of pulmonary arterial hypertension
}

Reply to F. Antigny and co-workers:

Mathias Declercq1,2,3,7, Lucas Treps2,3,4,7, Siham Bousfia1,2,3, Peter Carmeliet2,3,8 and Peter Witters $1,5,6,8$

1Dept of Development and Regeneration, CF Centre, Woman and Child, KU Leuven, Leuven, Belgium. 2Laboratory of Angiogenesis and Vascular Metabolism, Centre for Cancer Biology, VIB, Leuven, Belgium.

3Laboratory of Angiogenesis and Vascular Metabolism, Dept of Oncology and Leuven Cancer Institute (LKI), KU Leuven, Leuven, Belgium.

${ }_{4}$ Université de Nantes, CNRS, INSERM, CRCINA, Nantes, France.

5Dept of Paediatrics, University Hospitals Leuven, Leuven, Belgium.

${ }_{6}$ Centre of Metabolic Diseases, University Hospitals Leuven, Leuven, Belgium.

7 Equal co-authorship.

${ }_{8}$ Co-corresponding authorship.

F. Antigny and co-workers raise interesting points in their comment on the transcriptomic analysis of cystic fibrosis transmembrane conductance regulator (CFTR)-impaired endothelial cells (ECs) revealing a pro-inflammatory phenotype [1]. Indeed, CFTR impairment in different endothelial models and organisms, and in the absence of concomitant infection, suggests that ECs are an overlooked mediator in the exaggerated pro-inflammatory phenotype observed in cystic fibrosis (CF). Whether endothelial CFTR is involved in other vascular diseases is an intriguing question. Indeed, a recent report indicates a pivotal role of CFTR in pulmonary arterial hypertension (PAH) [2].

Different cell types are involved in the pathogenesis of PAH, namely ECs, vascular smooth muscle cells (vSMCs), fibroblasts and leukocytes. As recently reviewed by EVANS et al. [3], the role of ECs in PAH is crucial and multi-faceted. The endothelium is the conductor of numerous pathophysiological processes deregulated in $\mathrm{PAH}$, including vasoconstriction, inflammation, coagulation, oxidative stress, cell viability, metabolism, proliferation, migration and differentiation [3]. In our study, we found that CFTR impairment leads to a wide spectrum of overlapping endothelial malfunctions including increased EC activation with leukocyte extravasation, oxidative stress, reduced cell viability, growth, delayed wound repair and halted autophagy (figure 1) [1].

Extrapolating our findings and combining them with the recent study from LE RIBEUZ et al. [2] suggests that CFTR impairment is important in the pathogenesis of PAH. While endotheliumindependent relaxation is present upon CFTR dysfunction in animal models, there is clear evidence that endothelial CFTR could influence the vascular tone [4]. Descriptive in situ studies demonstrated reduced endothelium-dependent relaxation of human CF pulmonary arteries [5, 6]. Additionally, in the Framingham Heart Study, CFTR was associated with brachial artery flow-mediated dilation, which is a marker for the in vivo assessment of endothelial nitric oxide bioavailability, a key mediator of vascular function [7]. Finally, there is micro- and macrovascular dysfunction in CF patients, which could be partially restored by rescuing endothelial NO production with sildenafil, tetrahydrobiopterin and CFTR modulators (figure 1) [8,9]. Altogether, this demonstrates a role of endothelial CFTR in vasomotor regulation.

Histologically, PAH is characterised by the muscularisation of arterioles, intimal fibrosis and medial hypertrophy, and the hyperproliferation of ECs and vSMCs, as was found from CFTR-impaired models (figure 1) [2]. Multiple studies have also characterised endothelial-to-mesenchymal transition (EndMT) to be involved in the vascular remodelling in PAH [10]. EndMT is a process whereby ECs lose their key endothelial traits and start expressing mesenchymal markers. As such, EndMT may directly or indirectly contribute to $\mathrm{PAH}$, by EC transformation into smooth muscle-like cells with 
higher proliferative and migratory potential [10]. Accordingly, we recently underlined that various CFTR-impaired EC models showed limited EndMT, which might represent a secondary event due to CFTR-related endothelial dysfunction (figure 1). Interestingly, CFTR modulators were able to reduce the expression of mesenchymal markers, EC activation and its subsequent leukocyte adhesion [1, 11]. It remains to be demonstrated whether hypoxia or cytokine treatment could further trigger EndMT in CFTR-impaired ECs and thus play a role in the vascular remodelling observed in CF and PAH.

Altered autophagy and overproduction of reactive oxygen species (ROS) have been observed in lung diseases, including $\mathrm{CF}$ and $\mathrm{PAH}$ [10]. Moreover, increased shear stress is known to heighten oxidative stress and to activate autophagy. Increasingly, more evidence indicates that functional autophagy is necessary to maintain redox homeostasis [12]. We found increased total and mitochondrial ROS together with a loss in function autophagy in CFTR-impaired ECs (figure 1) [1]. The intricate interplay between autophagy, hypoxia, shear stress, EndMT and inflammation in CFTRimpaired EC models and the effect of CFTR modulators on the overproduction of ROS and restoration of autophagy in CF remains to be investigated.

Finally, an often overlooked factor in the regulation of the vascular tone is the vascular endothelialglycocalyx, a polysaccharide protein complex covering the surface of ECs. When the glycocalyx is damaged, it loses essential properties in regulating vascular permeability, anticoagulation, inflammation via immune cell-EC interactions and cytokine signalling, mechanotransduction and its role as a vasoprotective layer. This has been demonstrated in various pulmonary pathologies, for instance in COVID-19, where the endothelium plays a critical role [13]. Functional CFTR is required for endothelial glycocalyx swelling (figure 1) [14] and PAH could be attenuated by protecting the integrity of the endothelial glycocalyx in a rat monocrotaline-induced PAH model [15]. Hence, CFTR could be relevant to glycocalyx-healing strategies in lung diseases.

To conclude, the aforementioned findings further highlight that CFTR in ECs could play a key role in PAH, next to the vSMCs. As proposed by F. Antigny and co-workers, patient-derived ECs would be important to investigate; nevertheless, it is known that ECs change their phenotype after being cultured, making in vitro testing challenging [16]. Fundamental studies of CFTR-deficient cells under high shear stress and/or in presence of EndMT inducers may provide better understanding into the pathogenic mechanism of PAH. Finally, since multiple cell types are important for PAH pathogenesis, omics approaches, such as single cell RNA sequencing on patient tissue, will provide valuable insights to tackle this matter.

Author contributions: M. Declercq, L. Treps, S. Bousfia, P. Carmeliet and P. Witters wrote the manuscript. L. Treps

made the figure. All authors commented on the manuscript.

\section{Conflict of interest: None declared.}

\section{References}

1 Declercq M, de Zeeuw P, Conchinha NV, et al. Transcriptomic analysis of CFTR-impaired endothelial cells reveals a pro-inflammatory phenotype. Eur Respir J 2021; 57: 2000261.

2 Le Ribeuz H, To L, Ghigna M-R, et al. Involvement of CFTR in the pathogenesis of pulmonary arterial hypertension. Eur Respir J 2021; 57: 2000653.

3 Evans CE, Cober ND, Dai Z, et al. Endothelial cells in the pathogenesis of pulmonary arterial hypertension. Eur Respir J 2021; in press [https://doi.org/10.1183/13993003.03957-2020].

4 Declercq M, Treps L, Carmeliet P, et al. The role of endothelial cells in cystic fibrosis. J Cyst Fibros 2019; 18: 752-761. 
5 Xuan AT, Higenbottam TW, Pepke-Zaba J, et al. Reduced endothelium-dependent relaxation of cystic fibrosis pulmonary arteries. Eur J Pharmacol 1989; 163: 401-403.

6 Henno P, Maurey C, Danel C, et al. Pulmonary vascular dysfunction in endstage cystic fibrosis: Role of NF$\kappa \mathrm{B}$ and endothelin-1. Eur Respir J 2009; 34: 1329-1337.

7 Vasan RS, Larson MG, Aragam J, et al. Genome-wide association of echocardiographic dimensions, brachial artery endothelial function and treadmill exercise responses in the Framingham Heart Study. BMC Med Genet 2007; 8: Suppl. 1, S2.

8 Rodriguez-Miguelez P, Lee N, Tucker MA, et al. Sildenafil improves vascular endothelial function in patients with cystic fibrosis. Am J Physiol Circ Physiol 2018; 315: H1486-H1494.

9 Rink S, Janić M, Lunder M, et al. Effect of elexacaftor-tezacaftor-ivacaftor on pulmonary and endothelial function in a patient with advanced cystic fibrosis. ERJ Open Res 2021; 7: Suppl. 6, 104.

10 Gorelova A, Berman M, Al Ghouleh I. Endothelial-to-mesenchymal transition in pulmonary arterial hypertension. Antioxid Redox Signal 2020; 34: 891-914.

11 Treps L, Declercq M, Bousfia S, et al. Comparative meta-analysis of cystic fibrosis cell models suggests partial endothelial-to-mesenchymal transition. J Cyst Fibros 2021; in press [https://doi.org/10.1016/j.jcf.2021.03.019].

12 Liu J, Bi X, Chen T, et al. Shear stress regulates endothelial cell autophagy via redox regulation and Sirt1 expression. Cell Death Dis 2015; 6: e1827.

13 Teuwen L-A, Geldhof V, Pasut A, et al. COVID-19: the vasculature unleashed. Nat Rev Immunol 2020; 20: 389-391.

14 Peters W, Kusche-Vihrog K, Oberleithner H, et al. Cystic fibrosis transmembrane conductance regulator is involved in polyphenol-induced swelling of the endothelial glycocalyx. Nanomedicine 2015; 11: 1521-1530. 15 Guo J, Yang Z-C, Liu Y. Attenuating pulmonary hypertension by protecting the integrity of glycocalyx in rats model of pulmonary artery hypertension. Inflammation 2019; 42: 1951-1956.

16 Goveia J, Rohlenova K, Taverna F, et al. An integrated gene expression landscape profiling approach to identify lung tumor endothelial cell heterogeneity and angiogenic candidates. Cancer Cell 2020; 37: $21-$ 36.e13.

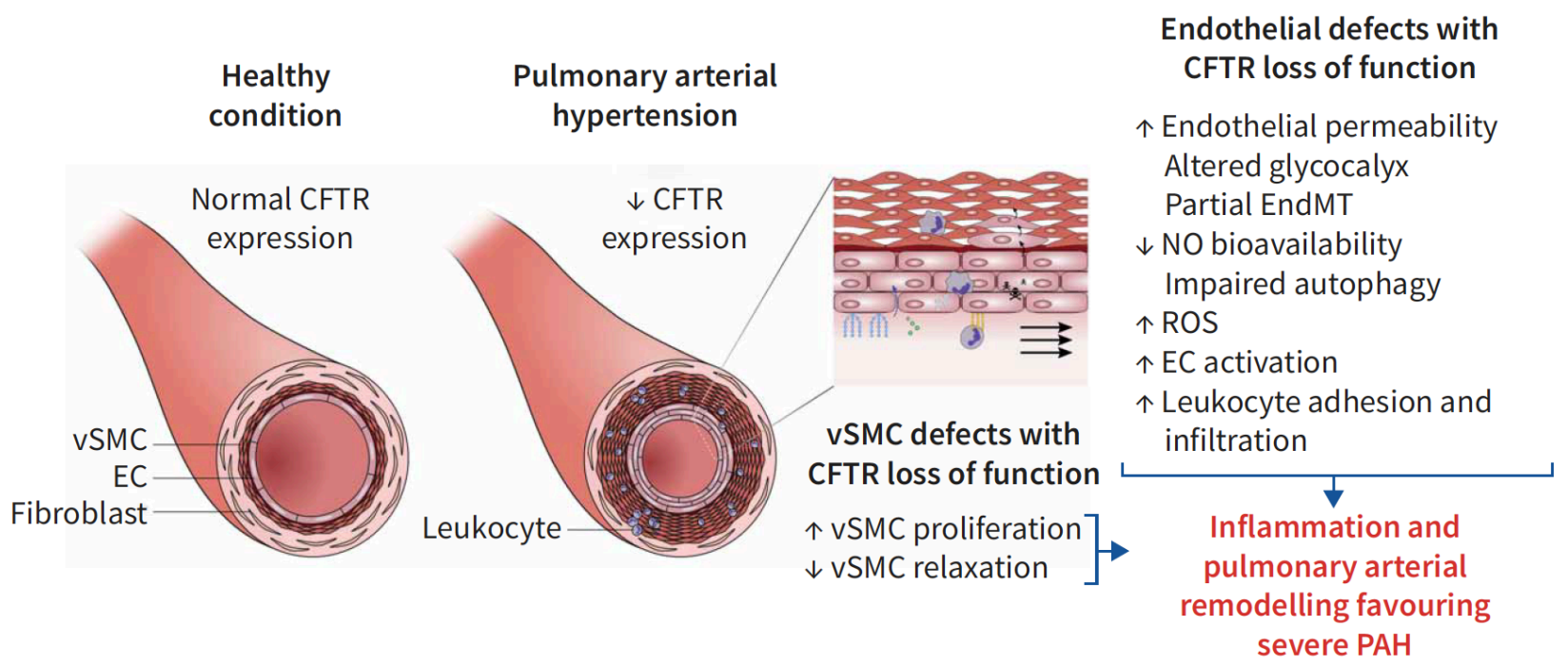

FIGURE 1 Proposed events arising from cystic fibrosis transmembrane conductance regulator (CFTR) dysfunction in pulmonary arterial hypertension (PAH) from the work done by DECLERCQ et al. [1], TREPS et al. [11], RODRIGUEZ-MIGUELEZ et al. [8] and LE RIBEUZ et al. [2]. vSMC: vascular smooth muscle cells; EC: endothelial cell; EndMT: endothelial-to-mesenchymal transition; NO: nitric oxide; ROS: reactive oxygen species. 